\title{
La motivation des décisions réglementaires et d'espèce en droit administratif français
}

\author{
Élise UNTERMAIER-KERLÉO \\ Maîtresse de conférences en droit public à l'université Jean-Moulin - Lyon 3 \\ Équipe de droit public de Lyon
}

I. Motivation et délibération

A. Rapports de présentation et notices explicatives des actes réglementaires nationaux, des ersatz de motivation

B. Des exigences plus poussées de motivation au niveau local

\section{Motivation et participation}

A. Une motivation en trompe-l'œil dans le champ de la démocratie environnementale

B. Des exigences de motivation édulcorées dans le cadre des autres procédures participatives

III. Motivation et évaluation

A. L'exigence élevée de motivation dans le cadre de l'évaluation environnementale

B. La fiche d'impact, substitut général de la motivation pour les décisions réglementaires et d'espèce

Avec la loi informatique et libertés du 6 janvier $1978^{1}$, suivie de celles du 17 juillet 1978 consacrant la liberté d'accès aux documents administratifs ${ }^{2}$ et du 3 janvier 1979 sur les archives ${ }^{3}$, la loi du 11 juillet 1979 relative à la motivation des actes administratifs et à l'amélioration des relations entre l'administration et le public a sérieusement ébréché le dogme du secret administratif et contribué à faire de la transparence un «droit de l'homme de la troisième génération ${ }^{4}$. Désormais abrogée, elle restera dans l'histoire du droit administratif français. Toutefois, contrairement à ce que son intitulé semblait indiquer, la loi du 11 juillet 1979 ne s'appliquait pas à l'ensemble des actes administratifs, mais visait uniquement les décisions administratives individuelles. Et encore, pas toutes. Loin

1. Loi $n^{\circ} 78-17$ du 6 janvier 1978 relative à l'informatique, aux fichiers et aux libertés.

2. Loi n ${ }^{\circ}$ 78-753 du 17 juillet 1978 portant diverses mesures d'amélioration des relations entre l'administration et le public et diverses dispositions d'ordre administratif, social et fiscal.

3. Loi no $79-18$ du 3 janvier 1979 sur les archives.

4. La formule, lancée par K. Vasak, «Pour les droits de l'homme de la troisième génération: les droits de solidarité» (leçon inaugurale des cours à l'Institut international des droits de l'homme, session de 1979), fut reprise par G. Braibant («À propos des lois françaises d'accès à l'information administrative", au colloque "Informatique et libertés», Fontevraud, mars 1979) et A. Holleaux ("Les lois de la "troisième génération des droits de l'homme": ébauche d'étude comparative», Revue française d'administration publique, n 15, juillet-septembre 1980, p. 527 ; «1978 An I de l'information administrative", Les petites affiches, ${ }^{\text {os }} 21 / 22,16$ et 19 février 1979). Pour une analyse critique de cette qualification, voir J.-F. Kerléo, La transparence en droit. Recherche sur la formation d'une culture juridique, Paris, Mare \& Martin, 2015, p. 719 sq. 
de consacrer une obligation générale ${ }^{5}$, ses dispositions, désormais reprises aux articles L. 211-2 à L. 211-7 du Code des relations entre le public et l'administration (CRPA), n'ont rendu la motivation obligatoire que pour certaines catégories de décisions individuelles défavorables à leurs destinataires (CRPA, art. L. 211-2). Autrement dit, certaines décisions, bien qu'elles soient défavorables à leurs destinataires, n'ont pas à être motivées. C'est le cas, par exemple, des décisions de mutation d'office des fonctionnaires ${ }^{6}$, ou encore des décisions qui refusent un avantage dont l'attribution ne constitue pas un droit, comme le refus opposé à une demande d'inscription en crèche municipale ou les décisions des fédérations sportives refusant de sélectionner des sportifs, en vue d'une compétition? ${ }^{7}$. Par ailleurs, échappent également à l'obligation de motivation les décisions individuelles, telles qu'un permis de construire ${ }^{8}$, qui sont favorables à leurs destinataires mais défavorables aux tiers ${ }^{9}$. Pour tenir compte de ces derniers, la loi de 1979 a uniquement prévu la motivation des décisions individuelles accordant des dérogations (CRPA, art. L. 211-3) ${ }^{10}$.

Ainsi la motivation, dans la loi de 1979, est-elle toujours destinée à une personne déterminée, en principe le destinataire de la décision qui lui est défavorable, voire le tiers à une décision accordant une dérogation à une autre personne. C'est ce qui pourrait expliquer que la motivation ait été jugée inutile tant pour les actes réglementaires, parce qu'ils s'appliquent à une catégorie de personnes (les mineurs, les étrangers, les automobilistes, etc.), que pour les actes d'espèce, qui visent une chose déterminée et unique (tel projet de construction, tel bâtiment, etc.). Mais cet argument n'est pas convaincant. Une norme, qu'elle soit générale ou d'espèce, s'adresse toujours, quoiqu'indirectement, à des personnes, auxquelles il peut être pertinent d'expliquer ses motifs. Ainsi, depuis $1966^{11}$, les arrêtés réglementaires que le maire peut adopter pour «[i]nterdire à certaines heures l'accès de certaines voies de l'agglomération" ou encore pour « [r]églementer l'arrêt et le stationnement des véhicules ou de certaines catégories d'entre eux» doivent être motivés ${ }^{12}$. S'est ainsi fait sentir la nécessité de justifier l'édiction d'une mesure réglementaire de police restreignant la liberté d'aller et venir. Le maire doit donc prendre soin, non seulement de citer les dispositions du Code l'habilitant à agir, mais encore de préciser les circonstances de fait justifiant l'application, dans sa commune, d'une interdiction de la circulation automobile à certaines heures ${ }^{13}$.

Quoi qu'il en soit, alors qu'elle aurait pu au moins, dans le prolongement de la loi de 1966 précitée, imposer la motivation de l'ensemble des règlements de police, la loi de 1979 a totalement exclu de son champ d'application les décisions réglementaires et les décisions d'espèce ${ }^{14}$. Les sénateurs s'en étaient émus, en tout cas pour les décrets réglementaires. L'absence de motivation de ce qu'ils appelaient à l'époque les «actes intermédiaires », c'est-à-dire les actes d'espèce, tels qu'une déclaration d'utilité publique ou les décisions en matière d'urbanisme, portant création de zones ou tendant à la protection de certains sites, ne présentait pas «de trop grands inconvénients car la plupart de ceux-ci sont actuellement précédés d'enquêtes qui offrent aux particuliers les moyens d'être informés sur les objectifs poursuivis par l'Administration ». En revanche, les sénateurs regrettaient «que les autorités qui, depuis 1958, disposent d'un pouvoir réglementaire autonome soient dispensées de motiver les décrets ", invitant le gouvernement à "s'engager plus systématiquement à faire précéder les décrets réglementaires d'un exposé des motifs qui en faciliterait grandement la compréhension et, par conséquent, l'application ${ }^{15}$.

5. Sur les critiques doctrinales quant au champ restreint de la motivation en droit français, voir notamment: J.-L. Autin, «La motivation des actes administratifs unilatéraux, entre tradition nationale et évolution des droits européens", Revue française d'administration publique, 2011, p. 85 sq.; G. Éveillard, «L'adoption des actes administratifs unilatéraux. Forme, délais, signature», Revue française de droit administratif, 2016, Le Code des relations entre le public et l'administration, p. 40 sq.; O. Gabarda, "Vers la généralisation de la motivation obligatoire des actes administratifs? Enjeux et perspectives d'évolutions autour du principe de la motivation facultative ", Revue française de droit administratif, 2012, p. 61 sq.; H. de Gaudemar, "L'obligation de motivation des actes administratifs unilatéraux en droit français", in La motivation en droit public, S. Caudal (dir.), Paris, Dalloz (Thèmes et commentaires), 2013, p. 69 sq.; I. Papadamaki, «L'obligation de motivation en droit administratif français sous l'influence du droit de l'Union européenne», Revue du droit public, 2017, p. 1245 sq.; É. Untermaier-Kerléo, «Regard critique sur le droit administratif français à l'aune de quelques exemples en droit comparé», in La motivation en droit public, p. 103 sq.

6. CE, 21 octobre 1983, Ministre de la Justice c. Poinçon, $\mathrm{n}^{\circ}$ 39921, Recueil Lebon, p. 419.

7. CE, 22 février 1991, M Mes Bensimon, Guyot et Kamoun, ${ }^{\circ}{ }^{\text {le }}$ 102775, Recueil Lebon, p. 61.

8. En revanche, le refus de permis doit être motivé, en application des dispositions spécifiques de l'article L. 424-3 du Code de l'urbanisme.

9. Voir, notamment, CE, sect., 9 décembre 1983, Vladescu, $\mathrm{n}^{\circ} 43407$, Recueil Lebon, p. 497, à propos du décret autorisant une personne à changer de nom et contesté par le père de cette dernière; CE, 30 décembre 2009, $M^{m e}$ Reilles et autres, $\mathrm{n}^{\circ}$ 297433, tables du Recueil Lebon, p. 636, concernant le décret autorisant une association à accepter le legs universel consenti en sa faveur et contesté par les membres de la famille du donateur.

10. Voir, par exemple, CE, 3 novembre 1982, $M^{\text {He }}$ Mugler, $\mathrm{n}^{\circ}$ 34461, tables du Recueil Lebon, p. 505 : la décision d'ouverture d'une officine de pharmacie par dérogation doit être motivée; CE, 26 mai 2014, Société IMS Health, n 354903, tables du Recueil Lebon, p. 496-667: les délibérations de la CNIL autorisant, par dérogation à l'interdiction posée au I de l'article 8 de la loi du 6 janvier 1978, certaines catégories de traitement de données sensibles sont au nombre des actes devant obligatoirement être motivés.

11. Loi no 66-407 du 18 juin 1966 complétant l'article 98 du Code de l'administration communale et relative aux pouvoirs de police conférés aux maires en matière de circulation, Journal officiel de la République française, 22 juin 1966, p. 5092.

12. CGCT, art. L. 2213-2 (ancien article 98 du Code de l'administration communale, devenu article L. 131-4 du Code des communes). Voir aussi les articles L. 2213-1-1, L. 2213-3 et L. 2213-4, qui permettent au maire, par "arrêté motivé», de prendre certaines mesures au titre de la police de la circulation et du stationnement.

13. CE, 8 juillet 1994, Association des loueurs de scooters saintois, $\mathrm{n}^{\circ} 132295$, inédit au Recueil Lebon.

14. La loi ne concerne pas non plus les actes non décisoires, dont il ne sera pas question dans cette étude.

15. J. Thyraud, Rapport fait au nom de la commission des lois constitutionnelles, de législation, du suffrage universel, du règlement et d'administration générale sur le projet de loi relatif à l’obligation de faire connaître les motifs des actes administratifs, Sénat, $\mathrm{n}^{\circ} 352$, 29 mai 1979, p. 16; voir aussi p. 24; en ligne: https://www.senat.fr/rap/1978-1979/i1978_1979_0352.pdf. 
Exclue de la loi de 1979, la motivation des décisions réglementaires et d'espèce n'a, depuis, jamais fait l'objet d'un texte général. En l'état actuel des choses, ces actes ne sont pas soumis à une obligation de motivation comparable à celle des décisions administratives individuelles, qui conduit à l'annulation pour vice de forme de l'acte non motivé ou insuffisamment motivé ${ }^{16}$. Toutefois, la motivation constitue une étape spontanée du processus normatif: les rédacteurs d'un projet de texte sont naturellement amenés à présenter les considérations de droit et de fait qui le justifient, à toutes les personnes associées, d'une manière ou d'une autre, à son adoption. La motivation des décisions réglementaires ou d'espèce s'est donc progressivement développée, sous la forme de rapports, notes, notices ou documents annexés au projet de texte, voire à l'acte final, et destinés à informer les personnes associées à son élaboration, qu'il s'agisse des membres d'une assemblée délibérante, du public, ou d'entités administratives appelées à se prononcer dans le cadre d'une évaluation préalable. Ainsi la motivation s'estelle imposée peu à peu, comme une garantie accessoire des procédures qui gouvernent l'élaboration des actes réglementaires ou d'espèce, qu'il s'agisse de préparer la délibération (I), faire participer le public (II), ou évaluer l'impact d'un projet de décision (III).

\section{Motivation et délibération}

Les rapports, notes ou notices de présentation qui accompagnent, au niveau national, les ordonnances, les décrets et certains arrêtés réglementaires, répondent à une exigence minimale de motivation (A). En revanche, au niveau local, la loi impose des obligations plus poussées, notamment à travers la note explicative de synthèse ou le rapport sur les affaires soumises à délibération, qui doivent être transmis aux membres des assemblées délibérantes, et plus encore avec les rapports de présentation, qui constituent un élément obligatoire des documents d'urbanisme (B).

\section{A. Rapports de présentation et notices explicatives des actes réglementaires nationaux, des ersatz de motivation}

Le projet d'acte réglementaire destiné à être soumis à une pluralité de personnes associées à son élaboration est naturellement précédé d'un exposé, d'un rapport ou d'une notice le présentant dans ses grandes lignes et justifiant les choix retenus par rapport aux objectifs visés.

À l'instar des projets et propositions de loi qui sont précédés de l'exposé de leurs motifs ${ }^{17}$, selon « une tradition républicaine qui a pour objet de présenter les principales caractéristiques de ce projet et de mettre en valeur l'intérêt qui s'attache à son adoption ${ }^{18}$, les ordonnances, qui constituent, avant qu'elles ne soient ratifiées par une loi, des actes administratifs réglementaires, sont publiées au Journal officiel, "accompagnées d'un rapport de présentation ${ }^{19}$. Comme l'exposé des motifs des projets et propositions de loi, ce rapport est dépourvu de valeur juridique $^{20}$. Présenté au président de la République en vue de l'examen d'une ordonnance en Conseil des ministres, il «a pour objet de l'éclairer sur les raisons pour lesquelles le texte est proposé et sur son contenu» et «ne saurait être regardé, quels qu'en soient les termes, comme une décision susceptible d'être déférée au juge de l'excès de pouvoir ${ }^{21}$.

En outre, la doctrine a déjà souligné le manque de précision des rapports accompagnant certaines ordonnances, qui, faute de travaux préparatoires, produisent «un droit sans mémoire et sans âme ${ }^{22}$ et privent

[...] l'interprète de la richesse des discussions, de l'exposé des opinions et arguments ainsi que des raisons ayant conduit le législateur à trancher en faveur de telle ou telle solution $^{23}$.

Ainsi a-t-il été suggéré d'adjoindre au texte de l'ordonnance des commentaires plus substantiels, par exemple sous la forme «d'explications sous articles, de manière à faire saillir les changements par rapport au droit existant ou le sens des formules retenues ${ }^{24}$.

16. La jurisprudence Danthony (CE, Ass., 23 décembre 2011, $\mathrm{n}^{\circ} 335033$, Recueil Lebon, p. 649) ne s'applique pas au défaut de motivation d'une décision individuelle. À la différence du vice de procédure, le défaut de motivation est un vice de forme qui entraîne systématiquement l'annulation de la décision attaquée. Par suite, le juge administratif n'a pas à rechercher si le vice de forme tenant à l'absence ou à l'insuffisance de motivation de la décision attaquée a été susceptible d'exercer une influence sur le sens de cette décision ou a privé le requérant d'une garantie (CE, 7 décembre 2016, Caisse d'assurance retraite et de santé au travail (CARSAT) d'Aquitaine, no 386304 , tables du Recueil Lebon, p. 610-864-903).

17. Pour les projets de loi, cette exigence figure expressément à l'article 7 de la loi organique $\mathrm{n}^{\circ} 2009-403$ du 15 avril 2009 relative à l'application des articles 34-1, 39 et 44 de la Constitution. Pour les propositions de loi, ce sont les Instructions générales du Bureau de l'Assemblée nationale (art. 3) et du Sénat (V) qui exigent qu'elles soient précédées d'un exposé des motifs.

18. CC, déc. $\mathrm{n}^{\circ}$ 2009-579 DC du 9 avril 2009, Loi organique relative à l'application des articles $34-1,39$ et 44 de la Constitution, cons. 11. Voir aussi, s'agissant de l'exposé des motifs envoyé aux électeurs, en même temps que le projet de loi soumis au référendum: CC, déc. n²005-33 REF du 7 avril 2005, Philippe de Villiers et Guillaume Peltier.

19. CRPA, art. L. 221-9.

20. En revanche, le rapport de présentation d'une ordonnance est publié au Journal officiel de la République française, alors que l'exposé des motifs d'un projet ou d'une proposition de loi ne l'est pas. Il est toutefois mis en ligne sur le site Légifrance, rubrique "Dossiers législatifs", dès le dépôt du projet de loi au Parlement et figure dans la version du projet mis en ligne sur le site de l'assemblée où le dépôt a été effectué.

21. CE, sect., 19 octobre 2005, CGT et autres, $\mathrm{n}^{\circ} 283471$, Recueil Lebon, p. 430.

22. P. Deumier, «Le Code civil, la loi et l'ordonnance (à propos du projet de loi relatif à la modernisation et à la simplification du droit et des procédures dans les domaines de la justice et des affaires intérieures du 27 novembre 2013, art. 3)», Revue trimestrielle de droit civil, 2014, p. 597 sq.

23. P. Puig, «L'autorité des rapports relatifs aux ordonnances (à propos du rapport au président de la République relatif à l’ordonnance n $2016-131$ du 10 février 2016 portant réforme du droit des contrats, du régime général et de la preuve des obligations) ", Revue trimestrielle de droit civil, 2017, p. 84 sq.

24. O. Deshayes, «Le droit des obligations est-il une chose trop grave pour être confiée à des ministères? ", Revue des contrats, $\mathrm{n}^{\circ}$ 110q2, 2014 , p. 157 
Jusqu'en 2011, les projets de décret - qu'ils aient une portée générale ou particulière -, ainsi que les projets d'arrêté soumis à la signature du Premier ministre, étaient, eux aussi, accompagnés d'un rapport de présentation, considéré comme l'équivalent de l'exposé des motifs d'un projet de $\operatorname{loi}^{25}$. Aux termes de la circulaire du 7 juillet 2011 relative à la qualité du droit (NOR: PRMX1118705C), une "notice explicative» s'est substituée au rapport de présentation pour les décrets réglementaires et certains arrêtés réglementaires, hormis les cas où celui-ci est prévu par un texte. Cette notice, qui accompagne le projet de texte dès sa rédaction, est publiée en même temps que ce dernier au Journal officiel. À l'instar de l'exposé des motifs d'un projet de loi et du rapport de présentation d'une ordonnance, la notice explicative est dépourvue de valeur juridique. Prévue par une simple circulaire, elle n'est pas «une condition de légalité des décrets réglementaires». Par conséquent, les requérants ne peuvent utilement invoquer, pour contester le décret attaqué, l'absence de publication d'une notice explicative ${ }^{26}$. Selon la circulaire de 2011, la notice explicative constitue "un document synthétique destiné à éclairer le lecteur du Journal officiel de la République française sur la portée du texte nouveau». C'est un document court (une demi-page), composé de plusieurs rubriques ("publics concernés», "objet», "entrée en vigueur», "notice», "références»), dont la «vocation est de donner une information fiable et accessible sur la nature et la portée des mesures susceptibles d'intéresser directement les destinataires des textes». Difficile donc, eu égard à sa concision et aux rubriques qui la composent, d'assimiler la notice explicative à la motivation exigée pour les décisions individuelles depuis la loi de 1979. En dépit des regrets exprimés par les sénateurs au cours des travaux préparatoires de cette dernière, les décrets réglementaires ne sont toujours pas précédés d'un exposé de leurs motifs.

\section{B. Des exigences plus poussées de motivation au niveau local}

Au niveau local, le droit des membres des assemblées délibérantes d'être informés des affaires de la collectivité se concrétise par l'envoi d'une "note explicative de synthèse sur les affaires soumises à délibération", adressée avec la convocation aux membres du conseil municipal dans les communes de 3500 habitants et plus (Code général des collectivités territoriales - CGCT, art. L. 2121-12), ainsi que dans les établissements publics de coopération intercommunale comprenant au moins une commune de 3500 habitants et plus (CGCT, art. L. 5211-1). De la même façon, douze jours au moins avant la réunion du conseil départemental ou régional, le président adresse aux conseillers départementaux ou régionaux un rapport, sous quelque forme que ce soit, sur chacune des affaires qui doivent leur être soumises (CGCT, art. L. 3121-19 pour les conseillers départementaux et art. L. 4132-18 pour les conseillers régionaux). La note explicative de synthèse remplit bien une fonction de motivation puisque, sans avoir à justifier de manière détaillée « du bien-fondé des propositions qui leur sont soumises», elle

[...] doit permettre aux intéressés d'appréhender le contexte ainsi que de comprendre les motifs de fait et de droit des mesures envisagées et de mesurer les implications de leurs décisions $[\ldots]^{27}$.

Toutefois, à la différence de la motivation qui, en application des dispositions issues de la loi de 1979, constitue un élément même de la décision finale, la note ou le rapport adressé aux membres des assemblées délibérantes interviennent en amont de la délibération qui sera finalement adoptée, et se limitent donc à présenter les motifs du projet de délibération. Par conséquent, alors que le non-respect de l'obligation de motivation des décisions individuelles constitue un vice de forme entachant systématiquement d'illégalité la décision prise, la fourniture de la note explicative de synthèse ou du rapport constitue une formalité obligatoire dont la méconnaissance, en application de la jurisprudence Danthony ${ }^{28}$,

[...] n'est de nature à entacher d'illégalité la décision prise que s'il ressort des pièces du dossier qu'il a été susceptible d'exercer, en l'espèce, une influence sur le sens de la décision prise ou qu'il a privé les intéressés d'une garantie $[\ldots]^{29}$.

Par ailleurs, depuis longtemps (depuis toujours?), les documents d'urbanisme comportent un rapport de présentation. Cette exigence, qui correspondait sans doute à une pratique spontanément suivie au sein des services administratifs chargés de l'élaboration de ces documents, est apparue expressément dans les décrets d'application de la loi no 67-1253 du 30 décembre 1967 d'orientation foncière ${ }^{30}$. Elle a, depuis lors, été consacrée au niveau législatif. À l'origine, le plan d'occupation des sols était «accompagné d'un rapport de présentation ${ }^{31}$. Désormais,

25. Voir la circulaire du 30 janvier 1997 relative aux règles d'élaboration, de signature et de publication des textes au Journal officiel et à la mise en œuvre de procédures particulières incombant au Premier ministre; puis la circulaire du $1^{\text {er }}$ juillet 2004 relative aux règles d'élaboration, de signature et de publication des textes au Journal officiel de la République française.

26. CE, 20 juin 2016, Association citoyenne intercommunale des populations concernées par le projet d'aéroport de Notre-Dame-des-Landes, $\mathrm{n}^{\circ}$ 400364, tables du Recueil Lebon, p. 838.

27. Voir notamment CE, 14 novembre 2012, Commune de Mandelieu-La-Napoule, $\mathrm{n}^{\circ} 342327$, tables du Recueil Lebon, p. 602-603.

28. CE, Ass., 23 décembre 2011, Danthony, n³35033, Recueil Lebon, p. 649.

29. CE, 17 juillet 2013, Société française du radiotéléphone (SFR) et autres, $\mathrm{n}^{\circ} 350380$, tables du Recueil Lebon, p. 875.

30. Voir le décret $n^{\circ} 69-551$ du 28 mai 1969 relatif aux schémas directeurs d'aménagement et d'urbanisme et aux schémas de secteur et le décret $\mathrm{n}^{\circ} 70-1016$ du 28 octobre 1970 relatif aux plans d'occupation des sols.

31. Art. 16 du décret $n^{\circ} 70-1016$ du 28 octobre 1970 relatif aux plans d'occupation des sols. 
le document d'urbanisme «comprend $»$ un tel rapport ${ }^{32}$. Ce dernier, dont le contenu s'est considérablement enrichi au fil des réformes, est donc devenu un élément obligatoire du document d'urbanisme, intégré à celui-ci au stade du projet arrêté par l'organe délibérant compétent. Si les indications contenues dans ce rapport sont inopposables lors de la délivrance d'une autorisation d'urbanisme, elles peuvent être prises en considération par le juge pour interpréter les dispositions du règlement du plan local d'urbanisme, lorsque cette interprétation ne ressort pas clairement de la seule lecture du texte de ces dispositions ${ }^{33}$.

Le rapport a pour fonction d'informer, d'une part, les membres de l'organe délibérant compétent pour arrêter le projet de document puis pour approuver ce dernier, ainsi que les personnes publiques appelées à donner leur avis sur le projet de document, et d'autre part, le public, dans le cadre de l'enquête publique. Aux termes de l'article L. 151-4, al. $1^{\text {er }}$ du Code de l'urbanisme, le rapport de présentation du plan local d'urbanisme «explique les choix retenus pour établir le projet d'aménagement et de développement durables, les orientations d'aménagement et de programmation et le règlement». En cas de révision, modification ou mise en compatibilité du plan, «le rapport de présentation est complété par l'exposé des motifs des changements apportés » (Code de l'urbanisme, art. R. 151-5). Ainsi le rapport de présentation s'impose-t-il aux auteurs du plan comme «une exigence comparable pour les décisions individuelles à celle de la motivation », selon les termes de Bruno Lasserre, dans ses conclusions sur l'arrêt Daniau, en $1985^{34}$. Ainsi les omissions ou les insuffisances du rapport de présentation peuvent-elles conduire à l'annulation de la délibération approuvant le plan local d'urbanisme ou sa révision. Il en a été ainsi, par exemple, s'agissant d'un rapport de présentation qui

[...] n'apporte aucune précision quant aux motifs ayant conduit à maintenir certains secteurs en espaces boisés classés et à en exclure d'autres, alors qu'il résulte d'un document versé aux débats par la communauté d'agglomération, non joint au dossier du plan local d'urbanisme, que la superficie des espaces boisés classés est passée de 411,6 à 265,1 hectares, soit une diminution de 146,50 hectares pour l'ensemble du territoire du plan et de 63,8 à 36 hectares pour la seule ville d'Angers $[\ldots]^{35}$.

A également été annulée la délibération approuvant la révision d'un plan local d'urbanisme qui avait pour objet principal d'interdire, sur $80 \%$ du territoire de la commune, l'implantation d'antennes-relais, sans que le rapport de présentation expose les raisons de ces modifications du document d'urbanisme. Ainsi le rapport de présentation des documents d'urbanisme peut-il être considéré comme une exigence sensiblement équivalente à celle de la motivation qui s'impose aux décisions individuelles depuis la loi de $1979^{36}$.

\section{Motivation et participation}

Il est indispensable que le public soit informé des motifs de la décision à l'élaboration de laquelle il est amené à participer. Toutefois, les dispositions du Code de l'expropriation et du Code de l'environnement imposent une obligation de motivation en trompe-l'œil pour les décisions ayant une incidence sur l'environnement (A). Dans le CRPA comme dans le CGCT, les exigences quant à l'information du public sur les motifs de la décision sont encore plus édulcorées (B).

\section{A. Une motivation en trompe-l'œil dans le champ de la démocratie environnementale}

La motivation permet au public, tout d'abord, de participer en connaissance de cause, et ensuite, de savoir dans quelle mesure les observations qu'il a pu formuler ont été prises en compte. La participation du public à l'élaboration d'une décision administrative implique qu'il soit suffisamment informé des considérations de droit et de fait qui justifient tant le projet qui lui est soumis, que la décision finalement adoptée. Ainsi, lorsqu'il demande qu'un projet soit déclaré d'utilité publique, l'expropriant adresse au préfet un dossier qui sera tenu à la disposition du public pendant la durée de l'enquête, comprenant obligatoirement «une notice explicative» (Code de l'expropriation, art. R. 112-4 et R. 112-5). Cette notice a pour objet de pallier le volume et la complexité de certains dossiers qu'un public non averti aurait bien des difficultés à comprendre. Elle «indique l'objet de l'opération et les raisons pour lesquelles, parmi les partis envisagés, le projet soumis à l'enquête a été retenu, notamment du point de vue de son insertion dans l'environnement» (art. R. 112-6). Ainsi, ayant envisagé, pour supprimer un passage à niveau, soit de réaliser un passage routier souterrain, soit de réaliser un pont-route au-dessus de la voie ferrée, soit enfin de procéder à l'enterrement de la voie ferrée pour la faire passer sous la chaussée de la rue, l'administration était tenue d'informer le public des motifs pour lesquels la première possibilité a été seule soumise à l'enquête et retenue. En raison du caractère incomplet de la notice, la déclaration d'utilité publique est annulée ${ }^{37}$.

32. Art. L. 141-2 du Code de l'urbanisme s'agissant du schéma de cohérence territoriale, L. 151-2 pour le plan local d'urbanisme et L. 161-1 pour la carte communale.

33. CE, 10 février 2016, SCI Porte de Noisy, no 383738, tables du Recueil Lebon, p. 990-995.

34. CE, 22 novembre 1985, Ministre de l'Urbanisme c. Daniau, $\mathrm{n}^{\circ}$ 59719, concl. B. Lasserre, Recueil Lebon, p. 342; La semaine juridique, édition générale, $\mathrm{n}^{\circ}$ 27, 2 juillet 1986, II, 20633.

35. TA Nantes, 21 avril 2009, nº 064265, L'actualité juridique. Droit administratif, 2009, p. 1905, note N. Wolff.

36. CE, 17 juillet 2013, Société française du radiotéléphone (SFR) et autres.

37. CE, 3 octobre 1990, Hello et autres, $n^{\circ} 94637$, tables du Recueil Lebon, p. 819. Voir aussi CE, 24 novembre 1982, Colcombet, $n^{\circ}$ o9673, Recueil Lebon, p. 404; CE, 14 novembre 1997, Groupement des riverains, agriculteurs propriétaires et particuliers, $\mathrm{n}^{\circ} 168223$, inédit au Recueil Lebon. 
Par ailleurs, pour répondre aux exigences du droit de l'Union européenne ${ }^{38}$ et du droit international ${ }^{39}$, et comme le préconisaient les auteurs du rapport présenté par le Conseil d'État, L'utilité publique aujourd'hui ${ }^{40}$, la loi du 27 février 2002 relative à la démocratie de proximité a renforcé les exigences de transparence concernant la déclaration d'utilité publique (DUP). En vertu de l'article L. 121-3 (article L. 11-1-2 de l'ancien Code), «la décision refusant de déclarer d'utilité publique la réalisation d'un projet ou d'une opération est motivée». En outre, aux termes de l'article L. 122-1 du Code de l'expropriation (ancien article L. 11-1-1-3), qui concerne spécifiquement les opérations ayant une incidence sur l'environnement ou le patrimoine culturel, «l'acte déclarant d'utilité publique l'opération est accompagné d'un document qui expose les motifs et considérations justifiant son utilité publique». Pour le professeur Hostiou, ce dernier article est

[...] une disposition "pot de fleurs», dont le seul et unique objet est de témoigner de l'absence de désintérêt de notre Droit vis-à-vis des contraintes communautaires mais ne vise aucunement à modifier de quelque manière que ce soit les relations entre l'Administration et le public ${ }^{41}$.

En effet, afin d'éviter un contentieux fondé sur l'absence de motivation, ce n'est pas la DUP elle-même qui doit être motivée, mais seulement un document qui l'accompagne. Le Conseil d'État a confirmé que lesdites dispositions «ne sauraient être interprétées comme imposant une motivation en la forme de la déclaration d'utilité publique qui serait une condition de légalité de cette dernière ${ }^{42}$. La circonstance que le document explicatif prévu par les dispositions précitées n'ait pas été joint à la DUP ${ }^{43}$, ou qu'il ne soit pas visé par la DUP ${ }^{44}$, est sans influence sur la légalité de celle-ci. Le Conseil d'État a également refusé de remettre en cause les dispositions de l'article L. 11-1-1-3 du Code de l'expropriation dans le cadre de la question prioritaire de constitutionnalité. L'absence d'obligation de motivation de l'acte déclarant d'utilité publique une opération de réalisation de travaux ou d'aménagements ne méconnaît pas les droits garantis par l'article 7 de la Charte de l'environnement d'accéder aux informations relatives à l'environnement détenues par les autorités publiques et de participer à l'élaboration des décisions publiques ayant une incidence sur l'environnement ${ }^{45}$.

S'agissant de la procédure de participation du public, applicable aux décisions publiques, autres que les décisions individuelles, ayant une incidence sur l'environnement lorsque celles-ci ne sont pas soumises à une procédure particulière, les dispositions de l'article L. 123-19-1-II, dernier alinéa du Code de l'environnement prévoient qu'

Au plus tard à la date de la publication de la décision et pendant une durée minimale de trois mois, l'autorité administrative qui a pris la décision rend publics, par voie électronique, la synthèse des observations et propositions du public avec l'indication de celles dont il a été tenu compte, les observations et propositions déposées par voie électronique ainsi que, dans un document séparé, les motifs de la décision.

Là encore, comme en matière d'expropriation, le législateur n'a pas imposé la motivation de la décision adoptée à l'issue de la procédure participative, se contentant d'exiger que les motifs figurent dans un document séparé. L'idéal eut été de rendre obligatoire la motivation, soit dans le corps même de la décision, soit dans un document l'accompagnant mais qui conditionnerait la légalité formelle de cette dernière. Cette motivation devrait également avoir pour fonction d'expliquer dans quelle mesure les observations du public auraient ou non été prises en compte.

En outre, la loi est encore moins exigeante pour les plus petites collectivités. Les communes de moins de 10 oo habitants et groupements de moins de 30 ooo habitants peuvent recourir à des modalités de participation

38. Voir l'article 9 de la directive 97/11/CE du 3 mars 1997 selon lequel les motifs et considérations ayant fondé les décisions prises sur certains projets publics ou privés doivent être mis à la disposition du public.

39. Voir l'article $6, \S 9$, de la Convention sur l'accès à l'information et la participation du public au processus décisionnel et l'accès à la justice en matière d'environnement, du 25 juin 1998, qui prévoit que les parties devront, une fois la décision prise sur un certain nombre de projets sensibles pour l'environnement dont il dresse la liste, en communiquer le texte au public «assorti des motifs et considérations sur lesquels ladite décision est fondée».

40. Conseil d'État, L'utilité publique aujourd'hui, Paris, La documentation française (Les études du Conseil d'État), 1999. Ceci étant, cette position n'était pas unanimement partagée par les membres du Conseil d’État. Par un arrêt du 2 juin 2003, Union fédérale des consommateurs "Que choisir » de Côte d'Or (tables du Recueil Lebon, p. 817; L'actualité juridique. Droit administratif, 2003, p. 1978, note R. Hostiou; Cahiers juridiques de l'électricité et du gaz, 2003, p. 630, concl. C. Maugüé; La semaine juridique, édition administrations et collectivités territoriales, $\mathrm{n}^{\circ} 50,8$ décembre 2003, 2106, note P. Billet), la haute juridiction a considéré que les dispositions de la directive 97/11/CE du Conseil en date du 3 mars 1997 , qui exigent que l'auteur de la déclaration d'utilité publique (DUP), une fois cette dernière prise, porte à la connaissance du public une information supplémentaire explicitant les motifs et considérations qui ont fondé cette décision, ne sauraient être interprétées comme imposant une motivation en la forme qui serait une condition de légalité de cette dernière. Le moyen tiré de ce que, faute d'avoir été modifié en ce sens dans le délai de transposition imparti, le Code de l'expropriation, sur le fondement duquel a été prise la DUP contestée, serait incompatible avec les objectifs de cette directive, ne saurait, par suite, être accueilli. Voir encore, dans le même sens, CE, sect. TP avis, 6 mars 2001, nº 365815 , Collectivités publiques et concurrence, Paris, La documentation française (Études et documents du Conseil d'État; 53), 2002, p. 106.

41. R. Hostiou, "Motivation" de la DUP et refus de transmission d'une QPC», Revue de droit immobilier, 2013, p. 143. Voir aussi L. Molinero, "La "pseudo-motivation" de la déclaration d'utilité publique», in Études offertes au professeur René Hostiou, Paris, Litec, 2008, p. 401-419.

42. CE, 25 avril 2007, Commune de Beauregard-de-Terrasson, $\mathrm{n}^{\circ}$ 283016, tables du Recueil Lebon, p. 895.

43. CAA Versailles, 10 mai 2007, Département de l'Essonne, nº o6VEo1636.

44. CE, 15 novembre 2006, Société Placoplâtre et Commune de Vandières, nº 285658, tables du Recueil Lebon, p. 908.

45. CE, 19 avril 2013, Pernet Earl Pernet, $n^{\circ}$ 364498, inédit au Recueil Lebon; CE, 26 décembre 2013, Pernet Earl Pernet, $n^{\circ} 364498$, inédit au Recueil Lebon, L'actualité juridique. Droit immobilier, 2014, p. 16, étude S. Gilbert; R. Hostiou, «Non-motivation de la DUP et constitutionnalité de l'article L. 11-1-1-3 du code de l'expropriation", Revue de droit immobilier, 2014, p. 154. 
alternatives, notamment par le recueil des observations du public sur des registres papier. La synthèse des observations du public est rendue publique, sans que la décision ait à être obligatoirement motivée (Code de l'environnement, art. L. 123-19-1-III). Dans les communes de moins de 2000 habitants, une simple réunion publique peut se substituer à ces modalités de participation (art. L. 12319-1-IV).

\section{B. Des exigences de motivation édulcorées dans le cadre des autres procédures participatives}

Les exigences de motivation sont quasiment inexistantes dans les procédures d' "association» du public régies par le Code des relations entre le public et l'administration. S'agissant des «consultations ouvertes » permettant de recueillir sur un site Internet les observations des personnes concernées et qui peuvent se substituer à la consultation d'une commission administrative préalablement à l'édiction d'un acte réglementaire ${ }^{46}$, la loi impose seulement à l'administration d'établir, «au terme de la consultation », "une synthèse des observations qu'elle a recueillies, éventuellement accompagnée d'éléments d'information complémentaires", et qui est rendue publique. En outre, aux termes de l'article R. 132-5, la publication de la décision d'organiser une consultation ouverte en application de l'article L. 132-1

[...] est assortie du projet d'acte concerné et d'une notice explicative précisant l'objet et le contenu de celui-ci ainsi que, en tant que de besoin, la ou les dates prévues pour l'entrée en vigueur des mesures envisagées.

Concernant les «autres consultations ouvertes sur internet» (art. R. 132-8 à R. 132-10) susceptibles d'être organisées préalablement à l'adoption d'un acte réglementaire, pas une once de motivation ne s'impose à l'administration.

En revanche, pour les enquêtes publiques qui ne relèvent ni du Code de l'expropriation, ni du Code de l'environnement, le CRPA est un peu plus exigeant. Il impose la constitution d'un dossier comprenant notamment

[...] une notice explicative, qui indique l'objet du projet et les raisons pour lesquelles, parmi les partis envisagés, le projet soumis à l'enquête a été retenu, notamment du point de vue de son insertion dans l'environnement. (CRPA, art. R. 134-22)

En outre, à l'issue de l'enquête, "le commissaire enquêteur ou le président de la commission d'enquête rédige un rapport énonçant ses conclusions motivées » (CRPA, art. R. 134-26).

Une copie du rapport dans lequel le commissaire enquêteur ou la commission d'enquête énonce ses conclusions motivées est déposée à la mairie de la commune où s'est déroulée l'enquête ainsi que dans la ou les communes sur le territoire desquelles l'opération projetée faisant l'objet de l'enquête doit avoir lieu. (CRPA, art. R. 134-28)

Enfin,

[...] si les conclusions du commissaire enquêteur ou de la commission d'enquête sont défavorables à l'opération projetée, le conseil municipal est appelé à émettre son avis par une délibération motivée dont le procès-verbal est joint au dossier transmis au préfet. (CRPA, art. R. 134-30)

Dans le champ de la démocratie locale, le Code général des collectivités territoriales prévoit qu' «un dossier d'information sur l'objet du référendum décidé par la collectivité territoriale est mis à disposition du public» (CGCT, art. LO 1112-8). Ce dossier comporte, outre le texte de la question à laquelle les électeurs sont appelés à répondre et le projet de délibération ou d'acte soumis à leur approbation,

[...] un rapport explicatif exposant les motifs et la portée du projet ainsi que, le cas échéant, les caractéristiques techniques et financières de sa réalisation. (CGCT, art. R. 1112-2, al. 4)

En revanche, s'agissant de la procédure de consultation locale, les exigences quant à l'information du public sur les motifs sont moindres, sans doute parce que celui-ci donne seulement son avis mais ne décide pas. Le Code énonce plus sobrement que le dossier d'information

[...] comporte notamment la délibération par laquelle la consultation a été décidée à laquelle sont annexées les observations formulées par les membres de l'assemblée délibérante à l'occasion de cette délibération, ainsi qu'une notice d'information sur l'objet de la consultation. (CGCT, art. R. 1112-8)

Participation ne rime donc pas exactement avec motivation en droit administratif français, tant s'en faut. Si l'exigence d'information du public sur les motifs du projet de décision est, en règle générale, consacrée par les textes, en revanche, tel n'est pas le cas de la motivation de la décision finalement adoptée, qui permettrait pourtant à ce dernier de savoir dans quelle mesure ses observations ont été prises en compte, et éventuellement de comprendre pourquoi elles ne l'ont pas été.

\section{Motivation et évaluation}

La motivation s'est imposée dans le cadre du processus d'évaluation environnementale qui précède l'adoption de certains textes normatifs (A). En dehors du champ du droit de l'environnement, l'évaluation préalable des projets de textes normatifs - et avec elle, la motivation - se généralisent peu à peu $(B)$. 


\section{A. L'exigence élevée de motivation dans le cadre de l'évaluation environnementale}

En droit de l'environnement, le processus administratif décisionnel a été "révolutionné» par l'étude d'impact ${ }^{47}$, née aux États-Unis en 1970 et rapidement importée en droit français, grâce à une initiative parlementaire, par la loi $\mathrm{n}^{\circ} 76-629$ du 10 juillet 1976 relative à la protection de la nature. Définie comme « un rapport d'évaluation des incidences sur l'environnement» (Code de l'environnement, art. L. 122-1-II), son but est de «contraindre les autorités publiques et les acteurs privés à changer de mentalité et d'attitude ${ }^{48}$, afin de prévenir les atteintes à l'environnement. Pourtant pionnière en matière d'étude d'impact, ce n'est que sous la pression de l'Union européenne ${ }^{49}$ que la France étendra l'obligation de réaliser une évaluation environnementale aux «plans et programmes susceptibles d'avoir des incidences notables sur l'environnement », qu'il s'agisse des documents de planification relevant du Code de l'environnement ayant une incidence notable sur l'environnement (Code de l'environnement, art. L. 122-4 à L. 122-11) ou de certains documents d'urbanisme (Code de l'urbanisme, art. L. 104-1 à L. 104-8).

La phase d'évaluation préalable de ces décisions comporte des exigences assez élevées en termes de motivation. L'article L. 122-1-1-I du Code de l'environnement impose à l'autorité compétente pour autoriser un projet soumis à évaluation environnementale, de prendre une décision «motivée au regard des incidences notables du projet sur l'environnement». Le même article précise in fine que «la décision de refus d'autorisation expose les motifs du refus, tirés notamment des incidences notables potentielles du projet sur l'environnement». En outre, aux termes de l'article L. 122-1-1-IV, lorsque la décision d'octroi ou de refus d'autorisation d'un projet soumis à évaluation environnementale a été prise, l'autorité compétente en informe le public, ainsi que l'autorité environnementale et les collectivités territoriales intéressées par le projet. Selon l'article L. 122-9 du Code de l'environnement, lorsque le plan ou le programme soumis à évaluation environnementale a été adopté, l'autorité qui l'a arrêté en informe le public et l'autorité environnementale, en mettant à leur disposition, non seulement le plan ou le programme, mais aussi «une déclaration» résumant la manière dont il a été tenu compte du rapport sur les incidences environnementales, ainsi que «les motifs qui ont fondé les choix opérés par le plan ou le document, compte tenu des diverses solutions envisagées », et enfin «les mesures destinées à évaluer les incidences sur l'environnement de la mise en œuvre du plan ou du programme».
Quant au rapport de présentation des documents d'urbanisme soumis à évaluation environnementale, il « expose les raisons pour lesquelles, notamment du point de vue de la protection de l'environnement, parmi les partis d'aménagement envisagés, le projet a été retenu » (Code de l'urbanisme, art. L. 104-4, $3^{\circ}$ ).

Imposée par le droit de l'Union européenne, l'autorité environnementale, entité administrative originale composée d'experts, dotée d'une certaine autonomie vis-à-vis de l'autorité administrative décisionnaire ${ }^{50}$, veille à ce que les projets de travaux, d'ouvrages ou d'aménagements et les instruments de planification, dès lors qu'ils sont susceptibles d'avoir des incidences sur l'environnement, fassent préalablement l'objet d'une évaluation environnementale de qualité. L'autorité environnementale exerce une double compétence: elle délivre des avis sur la qualité de l'évaluation environnementale des projets, plans, programmes ou documents qui y sont soumis de manière systématique; elle prend la décision de soumettre ou au contraire de dispenser d'évaluation environnementale ceux qui relèvent de la procédure «au cas par cas». Dans le cadre de l'examen au cas par cas, la décision de l'autorité environnementale de soumettre ou non à une évaluation environnementale le projet ou le document de planification est toujours motivée (Code de l'environnement, art. R. 122-3-IV et art. R. 122-18-III; Code de l'urbanisme, art. 104-32). En outre, l'absence de réponse de l'autorité environnementale au terme du délai imparti vaut obligation de réaliser l'étude d'impact ou l'évaluation environnementale. En effet, comme l'affirmait l'avocat général D. Ruiz-Jarabo, dans ses conclusions sur l'affaire Commission c. Italie du 10 juin 2004:

La décision administrative consistant à nier que, du fait de ses spécificités, un projet porte atteinte à l'environnement, exige une motivation. Selon la règle générale [...] tout ouvrage, avant d'être autorisé, doit être soumis à une évaluation de sa portée, de sorte que, si une opération déterminée n'entre pas dans le champ de cette exigence de par son caractère non nuisible, il est nécessaire d'indiquer les raisons qui conduisent à affirmer une telle chose ${ }^{51}$.

Ainsi la motivation s'est-elle imposée dans le cadre de l'évaluation environnementale, sous la pression du droit de l'Union européenne.

\section{B. La fiche d'impact, substitut général de la motivation pour les décisions réglementaires et d'espèce}

Venue du droit de l'environnement, l'évaluation préalable à l'adoption d'actes normatifs s'est imposée en dehors

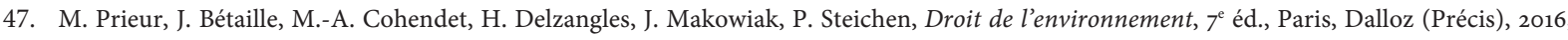
n 92, p. 111: l'étude d'impact est qualifiée de «procédure administrative révolutionnaire».

48. Ibid., n $\mathrm{n}^{\circ} 90$, p. 111.

49. Directive du 27 juin 2001 relative à l'évaluation des incidences de certains plans et programmes sur l'environnement (Parlement européen et Conseil de l'Union européenne, directive nº 2001/42/CE, 27 juin 2001, Journal officiel des Communautés européennes, L 197, 21 juillet 2001, p. 30 ).

50. É. Untermaier-Kerléo, «Une entité administrative originale: l'autorité environnementale», in L'environnementalisation du droit, Paris, Institut universitaire Varenne, à paraître.

51. CJCE, 10 juin 2004, Commission c. Italie, C-87/02, concl. D. Ruiz-Jarabo, point 36. 
du champ de celui-ci. C'est d'abord une circulaire du Premier ministre du 21 novembre 1995 qui a mis en œuvre, à titre expérimental, l'obligation d'assortir l'élaboration des projets de loi ou de décret réglementaire en Conseil d'État d'une étude ou fiche d'impact au champ élargi. Si l'exigence d'une étude d'impact a été consacrée dans la loi organique $\mathrm{n}^{\circ}$ 2009-403 du 15 avril 2009 relative à l'application des articles 34-1, 39 et 44 de la Constitution pour les projets de loi, c'est encore une simple circulaire, en date du 17 juillet 2013 relative à la mise en ouvre du gel de la réglementation (NOR: PRMX1318687C), qui impose une "fiche d'impact» pour tous les projets de textes réglementaires applicables aux entreprises ainsi qu'au public (particuliers, associations), à l'exclusion de ceux uniquement applicables aux administrations de l'État (textes d'organisation des services, dispositions à caractère budgétaire ou financier, règles applicables aux seuls agents de l'État). Une circulaire du Premier ministre du 12 octobre 2015 relative à l'évaluation préalable des normes et à la qualité du droit ( $\left.{ }^{\circ} 5817 / \mathrm{SG}\right)$ précise les seuils financiers au-delà desquels l'impact d'un projet de texte doit être en tout état de cause considéré comme significatif, pour les entreprises ou le public ${ }^{52}$. Une autre circulaire du 2 mai 2016 impose l'évaluation préalable de l'impact sur la jeunesse des projets de loi et de textes réglementaires.

S'agissant des projets de textes réglementaires créant ou modifiant des normes applicables aux collectivités territoriales et à leurs établissements publics, l'exigence d'une fiche d'impact a été consacrée au niveau législatif. L'article L. 1212-2 du Code général des collectivités territoriales impose que le Conseil national d'évaluation des normes (CNEN) soit consulté par le gouvernement sur l'impact technique et financier, pour les collectivités territoriales et leurs établissements publics, des projets de textes réglementaires créant ou modifiant des normes qui leur sont applicables. À cette fin, les projets de texte

[...] sont accompagnés d'un rapport de présentation et d'une fiche d'impact faisant apparaître les incidences techniques et les incidences financières, quelles qu'elles soient, des mesures proposées pour les collectivités territoriales ${ }^{53}$.

Par ailleurs, en cas d'extension des compétences d'une commune à un établissement public de coopération intercommunale, la loi (CGCT, art. L. 5211-4-1) a prévu préalablement à la décision de transférer les agents communaux, l'obligation d'établir une fiche d'impact, qui sera ensuite annexée à la décision, et décrivant notamment les effets du transfert sur l'organisation et les conditions de travail, ainsi que sur la rémunération et les droits acquis des fonctionnaires et des agents territoriaux non titulaires concernés.

En outre, conformément à l'article 8, II du décret $\mathrm{n}^{\circ}$ 2015-510 du 7 mai 2015 portant charte de la déconcentration,

L'ensemble des projets de texte réglementaire ayant des conséquences sur les missions et l'organisation des services déconcentrés de l'État font également l'objet d'une fiche d'impact préalable qui doit permettre de vérifier l'adéquation entre les objectifs poursuivis et les contraintes et moyens des services déconcentrés. Cette fiche d'impact est jointe aux projets dès leur transmission en vue des discussions interministérielles.

Les projets de décret en Conseil d'État sont transmis à celui-ci accompagnés de cette fiche d'impact.

Dans le même ordre d'idées, la création, par décret, d'une commission administrative consultative est

[...] précédée de la réalisation d'une étude permettant notamment de vérifier que la mission impartie à la commission répond à une nécessité et n'est pas susceptible d'être assurée par une commission existante ${ }^{54}$.

Bien qu'elle soit essentiellement réalisée dans le but de réduire l'inflation normative et limiter les dépenses publiques, l'évaluation préalable n'en constitue pas moins une forme de motivation. Une fiche d'impact comporte nécessairement une présentation générale du texte, de ses objectifs, c'est-à-dire les motifs de fait qui justifient l'adoption de l'acte normatif, ainsi qu'une rubrique «Environnement juridique» qui conduit à exposer les motifs de droit de l'acte. En l'état actuel des choses, l'exigence d'une évaluation préalable à l'adoption de décrets réglementaires ou d'espèce résulte de dispositions hétérogènes, dont la valeur normative est variable, et parfois nulle. Elle pourrait être généralisée, consacrée par des dispositions législatives insérées dans le CRPA et améliorée. À cet égard, plutôt que d'avoir à la rechercher dans la rubrique «Évaluation préalable des projets de norme» du site Légifrance, la fiche d'impact pourrait être publiée au Journal officiel avec le décret finalement adopté.

Certes, les décisions administratives réglementaires ou d'espèce ne sont pas soumises à une obligation de motivation comparable à celle qui s'impose aux décisions individuelles visées par la loi du 11 juillet 1979 et qui conduit à l'annulation pour vice de forme de l'acte non motivé ou insuffisamment motivé. Toutefois, l'exigence de motivation s'est développée de façon rampante: elle a pris la forme de

52. «Doivent en tout état de cause être regardés comme comportant un impact significatif sur les entreprises, les textes susceptibles d'entraîner une économie ou des coûts annuels de plus de 500 ooo euros pour l'ensemble des entreprises et de plus de 10 ooo euros pour au moins une entreprise. S'agissant des textes créant ou modifiant des normes applicables au public ou aux associations, est réputé comporter un impact significatif tout projet de texte entraînant une économie ou un coût annuel global de plus de 250 ooo euros ou de plus de 1000 euros pour un foyer ou pour une association".

53. CGCT, art. R. 1213-27, créé par décret nº 2014-446 du 30 avril 2014 portant application de la loi n $2013-921$ du 17 octobre 2013 portant création d'un Conseil national d'évaluation des normes applicables aux collectivités territoriales et à leurs établissements publics.

54. CRPA, art. R. 133-2, issu de l'art. 2 du décret $n^{\circ}$ 2006-672 du 8 juin 2006 relatif à la création, à la composition et au fonctionnement de commissions administratives à caractère consultatif, Journal officiel de la République française, 9 juin 2006, p. 8706. 
rapports, notes, notices, fiches ou documents annexés au projet de décision, voire à l'acte final, et destinés à informer les personnes associées à son élaboration, qu'il s'agisse des membres d'une assemblée délibérante, du public, ou d'entités administratives appelées à se prononcer dans le cadre de l'évaluation préalable des textes normatifs, telles que l'autorité environnementale ou le CNEN. La motivation des actes réglementaires ou d'espèce a longtemps été rejetée parce qu'elle ralentirait l'action administrative, constituant une charge supplémentaire pour les agents publics, aussi inutile que coûteuse. Cet argument ne tient plus: l'administration s'astreint déjà, en pratique, à la rédaction de rapports de présentation et de fiches d'impact chronophages. Il est donc urgent de compléter le CRPA par de nouvelles dispositions relatives à la motivation des décisions non individuelles. Il faut imposer, au minimum, une obligation de motivation pour toutes les mesures de police, qu'elles soient individuelles ou réglementaires, et faire précéder les décrets réglementaires d'un exposé des motifs. Il est grand temps d'insérer dans ce même Code un nouveau chapitre consacré à l'évaluation préalable, en passe de devenir une étape incontournable du processus administratif décisionnel, et pas uniquement dans le champ du droit de l'environnement. L'étude d'impact, que la France peut se flatter d'avoir introduite très tôt dans son système juridique avec la loi de 1976 sur la protection de la nature, a vocation à devenir un outil majeur de la procédure administrative non contentieuse, le vecteur de la transparence et de l'information en ce qui concerne l'élaboration des actes unilatéraux réglementaires ou d'espèce. 